\title{
ABORTION LAW OF NEPAL : A NEW ACHIEVEMENT
}

Nepal has one of the highest maternal mortality ratio in Asia. Unsafe abortion is one of the leading cause. Forty-six million women around the world have induced abortions every year. Developing world have $78 \%$ of induced abortion where as it is quite low (22\%) in developed world. Unsafe abortion seems to be very high, where abortion was not legalized. Unsafe abortion invites immediate as well as long term health problems. Most of the abortion related deaths are due to the complications of unsafe abortion. The international conference on population and development (ICPD) called for:

- Access to safe abortion services

- Access to compassionate

- $\quad$ Quality services for the complications arising from abortion

- $\quad$ Post abortion counseling and family planning service to reduce unwanted repeated pregnancies and repeated abortions.

His Majesty's Government announced the Nepal criminal code (Muluki Ain) on $1^{\text {st }}$ Chaitra 2058 (16 $6^{\text {th }}$ March, 2002) and Royal assent was given on $10^{\text {th }}$ Ashoj 2059 (27 ${ }^{\text {th }}$ September, 2002). The new law legalizes abortion under the following conditions:

- Up to 12 weeks of gestation on the request of the pregnant women.

- Up to 18 weeks of gestation in case of rape or incest.

- At any gestation if the pregnancy is harmful to the pregnant women's physical and mental health as certified by an expert physician.

- At any gestation if the fetus is suffering from a severely debilitating or fatal deformity as certified by an expert physician.

- Listed medical practitioners will provide comprehensive abortion care services.

- Only the pregnant woman holds the right to choose to continue or discontinue the pregnancy. If the pregnant woman is less than 16 years of age or not in a position to give consent (mentally incompetent), the nearest guardian or relative can give consent for abortion services.

- The law prohibits termination of pregnancy of any gestation for the sole purpose of sex selection.
This new achievement in the form of legalization of abortion will be instrumental in reducing maternal mortality in Nepal. In Nepal, 539 women per 100,000 line births die due to pregnancy and its complication. According to Ministry of Health, Maternal Mortality and Morbidity study of 1998, approximately $5.4 \%$ of all maternal deaths are due to abortion complications. WHO has estimated that worldwide 20 million unsafe abortion occur every year. 70,000 women die each year as a result of unsafe abortion and one in eight pregnancy related deaths are due to unsafe abortion.

Safe abortion needs good quality abortion services incorporating counseling training human source, infection prevention practices and adequate logistic support. Abortion is also supported by contraceptive services to prevent further unwanted pregnancy.

In Nepal, Comprehensive Abortion Care (CAC) programme is based on "Women Centered Model" developed by IPAS in Vietnam which supports an woman's ability to exercise sexual and reproductive health and rights.

CAC service is available in Maternity Hospital, Thapathali including 52 of 75 districts.

The major challenge is to expand CAC service to poor, rural and marginalized women. This requires definitive strategies to provide service to these communities. Services should be linked to primary health centers and the service providers should be well trained by the leading centers. Service charges to these communities should be subsidized or made free.

To minimize unsafe abortion which is still persisting in villages, messages about legalization of abortion should be well informed by media and women should be educated to seek services as early as possible. 